\title{
On invariant volumes of codimension-one Anosov flows and the Verjovsky conjecture
}

\author{
Masayuki Asaoka
}

Published online: 9 July 2009

(C) Springer-Verlag 2009

\section{Erratum to: Invent math (2008) 174: 435-462 \\ DOI 10.1007/s00222-008-0151-9}

In [1], the author claimed the solution to the Verjovsky conjecture, i.e., the complete classification of codimension-one Anosov flows up to topological equivalence in higher dimension. It was a consequence of the following three results which are mutually independent:

1. The main theorem of [1]: any codimension-one topologically transitive Anosov flow is topologically equivalent to a volume-preserving one.

2. Simić's theorem [2]: any volume-preserving codimension-one Anosov flow in higher dimension is topologically equivalent to the suspension flow of a hyperbolic toral automorphism.

3. Verjovsky's theorem [3]: any codimension-one Anosov flow in higher dimension is topologically transitive.

However, a serious gap was found in the proof of the Simićs theorem in [2] and it is not fixed yet. So, the Verjovsky conjecture is still open at this moment.

\section{References}

1. Asaoka, M.: On invariant volumes of codimension-one Anosov flows and the Verjovsky conjecture. Invent. Math. 174(2), 435-462 (2008)

2. Simić, S.N.: Volume preserving codimension one Anosov flows in dimensions greater than three are suspensions. Preprint, arXiv:math/0508024 [math.DS], 2005/2007

3. Verjovsky, A.: Codimension one Anosov flows. Bol. Soc. Mat. Mexicana (2) 19(2), 49-77 (1974)

The online version of the original article can be found under doi:10.1007/s00222-008-0151-9.

M. Asaoka ( $\bowtie)$

Department of Mathematics, Kyoto University, Kyoto 606-8502, Japan

e-mail: asaoka@math.kyoto-u.ac.jp 